\title{
Planar chiral metasurfaces with maximal and tunable chiroptical response driven by bound states in the continuum
}

\section{Tan Shi}

Jinan University

\section{Zi-Lan Deng}

Jinan University https://orcid.org/0000-0003-3861-6014

\section{GUANGZHOU GENG}

Institute of Physics

\section{Yixuan Zeng}

National University of Singapore

\section{Guangwei Hu}

National University of Singapore https://orcid.org/0000-0002-3023-9632

\section{Adam Overvig}

City University of New York

Junjie Li

Institute of Physics https://orcid.org/0000-0002-1508-9891

\section{Cheng-Wei Qiu}

National University of Singapore https://orcid.org/0000-0002-6605-500X

\section{Andrea Alu}

CUNY Advanced Science Research Center https://orcid.org/0000-0002-4297-5274

\section{Yuri S. Kivshar}

Australian National University

Xiangping Li ( $\nabla$ xiangpingli@jnu.edu.cn )

Jinan University https://orcid.org/0000-0003-0955-2613

\section{Article}

Keywords: Panar chiral metasurfaces, Bound states in the continuum, Extreme optical chirality, Circular dichroism, Chirality selectivity

Posted Date: January 26th, 2022

DOI: https://doi.org/10.21203/rs.3.rs-1194502/v1 
License: (c) (i) This work is licensed under a Creative Commons Attribution 4.0 International License. Read Full License

Version of Record: A version of this preprint was published at Nature Communications on July 15th, 2022. See the published version at https://doi.org/10.1038/s41467-022-31877-1. 


\title{
Planar chiral metasurfaces with maximal and tunable chiroptical response driven by bound states in the continuum
}

\author{
Tan Shi ${ }^{1, \dagger}$, Zi-Lan Deng ${ }^{1,+, *}$, Guangzhou Geng ${ }^{2, \dagger}$, Yixuan Zeng ${ }^{3}$, Guangwei $\mathrm{Hu}^{3}$, Adam Overvig ${ }^{4}$, \\ Junjie Lì ${ }^{2}$, , Cheng-Wei Qiu ${ }^{3}$, Andrea Alù ${ }^{4}$, Yuri S. Kivshar ${ }^{5}$, and Xiangping Li ${ }^{1, *}$
}

${ }^{1}$ Guangdong Provincial Key Laboratory of Optical Fiber Sensing and Communications, Institute of Photonics Technology, Jinan University, Guangzhou 510632, China

${ }^{2}$ Beijing National Laboratory for Condensed Matter Physics, Institute of Physics, Chinese Academy of Sciences, Beijing 100191, China

${ }^{3}$ Department of Electrical and Computer Engineering, National University of Singapore, Kent Ridge 117583, Republic of Singapore

${ }^{4}$ Photonics Initiative, Advanced Science Research Center, City University of New York, New York, NY 10031, USA

${ }^{5}$ Nonlinear Physics Center, Australian National University, Canberra ACT 2601, Australia

${ }^{\dagger}$ These authors contributed equally to this work

*E-mail: zilandeng@j,jnu.edu.cn; jili@iphy.ac.cn; xiangpingli@jnu.edu.cn

\begin{abstract}
:
Optical metasurfaces with high-Q chiral resonances can boost light-matter interaction for various applications of chiral response for ultrathin, active, and nonlinear metadevices. Usually, such metasurfaces require sophisticated depth-resolved nanofabrication to realize subwavelength stereonanostructures, posing overwhelming challenges, especially in the short-wavelength range. Here, we suggest a novel planarized design of chiral metasurfaces supporting bound states in the continuum (BICs), and demonstrate experimentally chiroptical responses with record-high Qfactors $(\mathrm{Q}=390)$ and near-perfect circular dichroism $(\mathrm{CD}=0.93)$ at optical frequencies. The symmetry-reduced meta-atoms are highly birefringent and support winding elliptical eigenpolarizations with opposite helicity surrounding the BIC polarization singularity, providing a convenient way for achieving maximal planar chirality tuned by either broken in-plane symmetry or changing illumination direction. Such sharply resonant chirality realized in planar metasurfaces promises various practical applications in classical and quantum optics including chiral sensing, enantiomer selection, and chiral quantum emitters.
\end{abstract}

Keywords: Planar chiral metasurfaces; Bound states in the continuum; Extreme optical chirality; Circular dichroism; Chirality selectivity 


\section{Introduction}

Chirality refers to the property of an object that cannot be superimposed with its mirror image after rotations or translations ${ }^{1-3}$. It is quite common in nature, e.g., in organic molecules ${ }^{4}$, quartz crystals ${ }^{5}$, and many others. The study of chirality is fundamentally important in various areas, including analytical chemistry, pharmaceutics, and even searching for extraterrestrial life. Light interactions with these geometries can induce chiroptical effects, including circular dichroism (CD) and optical activity, manifested by a difference in intensity and phase responses between left/right circularly polarized (LCP/RCP) light illuminations. Optical chirality was first demonstrated in three-dimensional (3D) photonic structures ${ }^{6}$, such as the helices ${ }^{6,7}$, twisted cross structures ${ }^{8,9}$ and multi-layered structures ${ }^{10-12}$, all exhibiting strong $\mathrm{CD}$ and optical activity with preserved circular polarization, which however require very demanding 3D nanofabrication techniques. Recently, twodimensional (2D) or planar structures have been shown to support both intrinsic planar chirality at normal incidence ${ }^{13-16}$ and extrinsic chirality at oblique incidences ${ }^{17-21}$. Being different from 3D chirality with broken mirror symmetry in the propagation direction, although even existing in some stacked structures ${ }^{15}$, planar chirality shows a circular polarization conversion between the output and incident light, and the cross-polarization behavior can be further exploited to modulate the geometric phase for arbitrary chiral wavefront shaping ${ }^{22-26}$. However, based on existing approaches it is challenging to achieve maximal planar chirality combined with ultra-high quality (Q-) factor due to absorption and scattering loss, hindering many applications that rely on strong chiral lightmatter interactions.

Importantly, bound states in the continuum (BICs) can provide a feasible solution for the outstanding problems of chiral photonics. BICs are identified as localized states coexisting with extended modes within the light cone, and they have attracted tremendous attention due to their unbounded Q-factors, which may boost light-matter interactions for applications such as surfaceemitting lasing ${ }^{27-29}$, biomedical sensors ${ }^{30-32}$, and nonlinear frequency conventers ${ }^{33-38}$. BICs originate from destructive interference of several radiative channels, and hence they cannot be accessed externally. Quasi-BICs (q-BICs) with high-Q resonances are tailored perturbing the symmetry to couple out the resonant mode to free-space radiation ${ }^{39-49}$. Recently, chiroptical nanostructures mediated by BICs have been proposed, demonstrating perfect unitary chirality and extremely high Q-factors ${ }^{50,51}$, and greatly expanding the available platforms to achieve optical chirality ${ }^{51-56}$. However, such chiral q-BIC scheme has only been proposed by complicated stereo-nanostructures, making elusive an experimental realization and application development, especially at optical frequencies.

Here, we suggest and realize experimentally a novel design of planar chiral metasurfaces at optical frequencies with simultaneously mediated maximal chirality and ultrahigh Q-factors. A double-sided scythe (DSS-) shaped $\alpha$-Si inclusion with in-plane inversion $\mathrm{C}_{2}$ symmetry but without in-plane mirror symmetry is employed to construct the BIC state. Such BIC state with strong birefringence supports a vortex polarization singularity ( $V$ point) surrounded by elliptical eigenstate polarizations with nonvanishing helicity, providing a convenient way to achieve maximum and 
high-Q chirality by slightly perturbing the inversion symmetry. With either breaking in-plane inversion symmetry or varying illumination geometry, we realize planar chiral q-BIC states with extremely strong intrinsic or extrinsic chirality, exceeding $\mathrm{CD}=0.99$ (in theory) and $\mathrm{CD}=0.93$ (in experiment). For the intrinsic chirality, the Q-factor of the $\mathrm{CD}$ spectra has an inverse quadratic relation with respect to the geometrical asymmetry, with a nearly unchanged CD peak. For the extrinsic chirality, both value and sign of $\mathrm{CD}$ can be flexibly tuned by changing the incident angles encircling the $\Gamma$ point, which is connected with the intrinsic nonvanishing helicities of the eigenpolarizations near the BIC singularity ${ }^{57}$. We believe that the demonstrated planar chiral metasurfaces governed by the BIC physics may find many applications in chiral sensing of biomolecules, spinselective emitters, and active chiroptical devices.

\section{Results and Discussion}

Previous q-BIC responses in metasurfaces usually did not support polarization effects ${ }^{28,43,44}$ (Fig. 1a) or only supported linear polarization selectivity ${ }^{30,39,41}$. Although very recently q-BICs with versatile polarization responses (chirality selectivity) were theoretically proposed, they required complicated 3D structures with broken symmetry in the propagation direction ${ }^{50,51,58}$ (Fig. 1b), hindering their implementation within current 2D patterning technology, especially at optical frequencies. Our present work shows that planar metasurfaces with only reduced in-plane symmetry can support q-BICs with both extrinsic and intrinsic planar chirality (Fig. 1c). Figure 1d shows the geometry of interest, a double-sided scythe (DSS) $\alpha$-Si structure (refractive indexes refer to Supplementary Fig. S2) employed as the unit cell of our proposed planar metasurface. Such DSS structure ( $W_{1}=W_{2}$ and $L_{1}=L_{2}$, but $\left.L_{1} \neq W_{1}\right)$ has in-plane inversion $\left(\mathrm{C}_{2}\right)$ symmetry, but lacks any inplane mirror symmetries. It hosts a BIC at the $\Gamma$ point, characterized by a vertical magnetic dipole (MD) mode as shown in Figs. 1(e and f). Different from the $\Gamma$ point BIC state supported by highly symmetric inclusions ${ }^{57}$, which is companied by a polarization singularity surrounded by vortex linear polarizations, the BIC state of the DSS structure supports a polarization singularity enclosed by a vortex elliptical eigen-polarization with non-vanishing helicity (see the inset of Fig. If and Supplementary Fig. S3). The planar chirality behavior can be predicted by temporal coupled mode theory (TCMT) (Supplementary Notes I \& II), which suggests that a perfect planar chiral response can be obtained by tuning the local response to be birefringent using a geometry that has an inversion center, and then breaking inversion symmetry with a suitable perturbation to yield an intrinsic planar chiral q-BIC. Our proposed DSS inclusion with reduced symmetry provides a smart perturbation degree of freedom along the $45^{\circ}$ direction, capable of achieving both maximal extrinsic and intrinsic chirality by perturbing a single parameter. As a general remark, in our systems the sign of the $\mathrm{CD}$ is determined by the sign of the helicity, while the maximum of CD does not necessarily coincide with circular eigenpolarization with the highest helicity, because there is non-zero background scattering accumulated by Jones matrix elements (see Supplementary Notes I and Supplementary Fig. S4).

Based on such BIC state supported by DSS inclusion with reduced symmetry, one can expect both extrinsic and intrinsic planar chirality by slightly breaking the BIC symmetry. Figures 2(a-d) 
show the q-BIC with near-unity extrinsic chirality achieved by illumination symmetry breaking (varying incident angle $\theta$ and conical angle $\varphi$ ). Here, the $\mathrm{CD}$ is defined as the transmittance difference under right-handed polarization (RCP) and left-handed polarization (LCP) incidence:

$$
C D=\frac{\left(T_{r r}+T_{l r}\right)-\left(T_{r l}+T_{l l}\right)}{\left(T_{r r}+T_{l r}\right)+\left(T_{r l}+T_{l l}\right)},
$$

where $T_{i j}=\left|t_{i j}\right|^{2}(i=r, l ; j=r, l ; r$ represents RCP, $l$ represents LCP $)$ is the transmittance of output polarization $i$ from the input polarization $j$, and all element of $t_{i j}$ construct the Jones matrix under circular polarization basis ${ }^{11,59}$

$$
J_{\text {circ }}=\left(\begin{array}{ll}
t_{r r} & t_{r l} \\
t_{l r} & t_{l l}
\end{array}\right)
$$

Figure $2 b$ shows the calculated transmission spectra of all Jones matrix elements and the $C D$ spectrum of the DSS metasurface with $\mathrm{C}_{2}$ symmetry $\left(W_{1}=W_{2}\right.$ and $L_{1}=L_{2}$, but $\left.L_{1} \neq W_{1}\right)$ at oblique incidence $\left(\theta=8^{\circ}, \varphi=90^{\circ}\right) . T_{l r}$ has a sharp peak greater than 0.9 , while all the other three Jones matrix elements ( $T_{r l}, T_{l l}$ and $T_{r r}$ ) exhibit dips close to 0 at the resonant wavelength $(1456 \mathrm{~nm})$ of the q-BIC, resulting in an ultra-sharp $C D$ spectrum with a maximum greater than 0.95 (green curves in Fig. 2b). Figure 2c shows the evolution of CD spectra by continuously varying the incident angle $\theta$ along the $y$-direction $\left(\varphi=90^{\circ}\right)$ (evolutions of transmission spectra of all Jones matrix elements are shown in Supplementary Fig. S5). The linewidth of the CD spectrum increases from 0 to finite values as $\theta$ increases, and the peak CD sustains near-unitary value with finite linewidths, manifesting the transfer process from a non-radiative BIC to a high-Q radiative $\mathrm{q}-\mathrm{BIC}$ with strong extrinsic chirality. The Q-factor of the extrinsic chiral q-BIC shows the expected inverse quadratic law with the illumination asymmetry parameter $\alpha_{l}=\sin \theta$ (Fig. 2d), which provides a convenient way to tailor the $\mathrm{Q}$-factor of the chiral response. The evolutions of the extrinsic chiral q-BIC along the $x$-direction $\left(\varphi=0^{\circ}\right)$ are shown in Supplementary Fig. S6, the variation trend is the same as the $\varphi=90^{\circ}$ case, except that the sign of CD is flipped due to the opposite helicity of eigen-polarization supported by the DSS structure along $x$ - and $y$-directions (the inset of Fig. 1f). To investigate the full CD flip picture, the evolution of CD spectra with varying conical angle $\varphi$ at a fixed incident angle $\theta=12^{\circ}$ is shown in Supplementary Fig. S7. The opposite varying trend of $T_{l r}$ and $T_{r l}$ can be observed as $\varphi$ is varying, leading to the sign flip of $\mathrm{CD}$ at positions near $(2 \mathrm{n}-1) \times 45^{\circ},(\mathrm{n}=1,2,3,4)$, which is consistent with helicity sign flip shown in the inset of Fig. 1f.

Beyond the extrinsic chiral behavior, we can also access intrinsic high-Q chiral behavior under normal incidence. This can be achieved by introducing an in-plane geometrical asymmetry parameter $\left(\delta=W_{2}-W_{1}\right)$ as shown in Fig. 2e. As the DSS structure supports strong birefringence along $45^{\circ}$, an in-plane perturbation that breaks inversion symmetry will provide a degree of freedom to obtain planar chirality, as TCMT suggests in supplementary Note II. Figure $2 \mathrm{f}$ depicts the transmission spectra of all Jones Matrix elements and the CD spectra of the metasurface with $\delta=40$ $\mathrm{nm}\left(W_{l}=191 \mathrm{~nm}, W_{2}=231 \mathrm{~nm}\right)$. The ultra-sharp resonant peak of $T_{l r}$ and resonant dips of $T_{r l}, T_{l l}$ and $T_{r r}$ are obtained at the wavelength of $1392 \mathrm{~nm}$, resulting in a CD peak greater than 0.99 and an ultranarrow linewidth of $1.45 \mathrm{~nm}$. We also confirm the planar chiral q-BIC modes by utilizing multipole 
expansions and studying the nearfield electromagnetic patterns of the modes (Supplementary Fig. S8), which further confirm that the high-Q planar chiral q-BIC states are dominated by the MD mode. Figures $2(\mathrm{~g}-\mathrm{h})$ show the $\mathrm{CD}$ evolution spectra as a function of the asymmetry parameter $\delta$, the Q-factor of the $\mathrm{CD}$ spectrum also manifests an inverse quadratic relationship against the relative asymmetry parameter defined by $\alpha_{2}=\delta / W_{l}$, which is the signature of symmetry-protected BICs (corresponding spectra for all components of the Jones matrix spectra refer to Supplementary Fig. S9). Different from the intrinsic 3D chirality with preserved helicity between input and output polarizations, the intrinsic planar chirality is always accompanied by circular conversion dichroism (CCD) and asymmetry transmission (AT) ${ }^{2,13,60}$, as shown in Supplementary Fig. S10, due to the preserved symmetry in the propagation direction. The CCD is produced by the mutual orientation of chiral elements and light transmission direction, and the peak value of CCD is close to 1, which is slightly larger than CD (Supplementary Fig. S11). Perfect unitary circular conversion transmittance $T_{l r}$ and CD can be achieved with a strictly symmetric background as shown in Supplementary Fig. S12. The existence of a practical $\mathrm{SiO}_{2}$ substrate introduces a perturbation of the background scattering, resulting in reduced transmittance and CD, as presented in Figs. 2(b and f).

Due to the symmetry-protected origin of this phenomenon, our designed DSS metasurface has good tolerance against fabrication imperfections, as tested in Supplementary Fig. S13, which provides a convenient way for experimental realization. We fabricated the DSS metasurfaces with different in-plane asymmetry parameters ( $\delta=0,20,40,60$, and $80 \mathrm{~nm})$, and employed a homemade optical setup (Fig. 3a) to measure the Jones matrix elements of the metasurface with a rotating stage. We first examine the extrinsic chiral q-BIC by measuring the DSS metasurface without in-plane geometry asymmetry $(\delta=0)$ for varying incident angles (Fig. 3b). Figure 3c shows the comparison between simulated and measured transmission Jones matrix spectra at different incident angles $\left(\theta=0^{\circ}, \pm 4^{\circ}, \pm 8^{\circ}, \pm 12^{\circ}\right)$ along the $\varphi=90^{\circ}$ direction. At normal incidence (middle panel of Fig. $3 \mathrm{c}$ ), all transmission spectra are smooth without resonant features, because the BIC state at the $\Gamma$ point is decoupled from any scattering channels of the external environment. At other incident angles, sharp circular conversion peaks of $T_{l r}$ and resonant dips of other three Jones matrix elements $\left(T_{l l}, T_{r l}, T_{r r}\right)$ emerge in both simulations and experiments. The measured absolute peak transmittances are greater than 0.78 for all incident angles, which is a little below the simulated 0.9 , mainly due to the practical scattering loss of the fabricated sample as well as a limited collection efficiency of the measurement setup. As expected, we can clearly observe the linewidth increment of the q-BIC peak with increasing incident angle in the experiment, consistent with our theory and simulations. From the measured Jones matrix spectra, we extracted the CD spectra for different incident angles corresponding to Eq. (1) in Fig. 3d. As we can see, sharp CD peaks appear at the q-BIC resonant wavelengths, the simulated/measured CD maxima reach $0.8 / 0.72,0.95 / 0.82$, and $0.96 / 0.88$ with the simulated/measured Q-factors of $616 / 258,143 / 96$, and 58/39 for incident angles $\theta= \pm 4^{\circ}, \pm 8^{\circ}, \pm 12^{\circ}$, respectively, while for normal incidence, the $\mathrm{CD}$ is always 0 as the $\mathrm{BIC}$ state is not accessed by external excitations. We also experimentally characterized the extrinsic chiral q-BIC along the $\varphi=0^{\circ}$ direction in supplementary Fig. S14 where sharp transmission peaks of $T_{r l}$ are observed, achieving 
opposite signs of simulated/measured CD: $-0.89 /-0.82,-0.95 /-0.88$, and $-0.97 /-0.91$ with the simulated/measured Q-factors of 798/312, 196/102, 85/55 at incident angles $\theta= \pm 4^{\circ}, \pm 8^{\circ}, \pm 12^{\circ}$, respectively, which further confirms the CD flip behavior.

To further experimentally verify the intrinsic chiral q-BIC, we characterized the DSS metasurfaces with different in-plane geometrical asymmetries, as shown in Fig. 4. Figure 4a shows the scanning electron microscope (SEM) images of fabricated samples with different asymmetry parameters $\delta$. From both the top view and slant view, we see that the fabricated DSS silicon pillars are smooth and uniform, in agreement with the theoretical design. Figures 4(b and c) show the simulated and measured transmission Jones matrix and CD spectra of the five fabricated metasurfaces. When $\delta=0$ (lowest panel in Figs. $4 \mathrm{~b}$ and $4 \mathrm{c}$ ), there is no resonance with $T_{r l}=T_{l r}$ and $T_{l l}=T_{r r}$ due to reciprocity, and $\mathrm{CD}=0$ crossing the whole frequency band. As the asymmetry parameter $\delta$ became non-zero, sharp circular conversion transmission peaks of $T_{l r}$ and CD appear, and the bandwidth increases with the increase of the asymmetry parameter (upper four panels in Figs. $4 \mathrm{~b}$ and $4 \mathrm{c}$ ). The simulated/measured CD maxima for the intrinsic planar chirality are $0.98 / 0.88$, $0.99 / 0.93,0.99 / 0.92$, and $0.99 / 0.91$ with the simulated/measured Q-factors of 2457/390, 480/121, $198 / 62$, and $128 / 38$ for $\delta=20,40,60$, and $80 \mathrm{~nm}$, respectively. Such simultaneously achieved strong chirality and high Q-factors in experiment promise chiroptical functionalities with strong lightmatter interactions.

\section{Conclusions}

We have proposed and experimentally realized planar chiral metasurfaces with in-plane symmetry-reduced inclusions. Such metasurfaces are governed by the BIC physics with birefringence degrees of freedom, identified as a vortex polarization singularity surrounded by ellipse eigenstate polarizations with non-vanishing helicity. Both extrinsic and intrinsic planar chirality can be realized by symmetry breaking. By illumination symmetry breaking, the BIC state can be transferred to q-BIC with strong extrinsic chirality accompanied by tunable linewidth and signs. By introducing in-plane structure asymmetry, the intrinsic planar chirality can be achieved under normal incidence with maximum CD of 0.99 (theory) and 0.93 (experiment) and Q-factors exceeding 390 (experiment) at optical frequencies. Beyond the chirality based on circular polarizations, we can achieve any elliptical chirality by varying simultaneously the degree of birefringence and the axis angle of the anisotropic inclusion, which suggests a way to spark new research works. Due to the high Q-factor of the planar chiral q-BIC with its accessibility and controllability, our results provide unique opportunities for many applications requiring ultrahigh Q-factors and chirality control, including circular polarization emitter, arbitrary wavefront lasing, and chiral nonlinear optics.

\section{Methods}

Simulation of reflection and transmission efficiency: Rigorous coupled wave analysis $\left(\mathrm{S}^{4}\right)^{61}$ simulations were carried out to simulate the planar chiral q-BIC metasurface and to calculate 
efficiencies for the co-polarization handedness-preserved coefficients, and the circular crosspolarization conversion coefficients transmission spectra. The metasurfaces are designed by $\alpha$-Si columns based on a $\mathrm{SiO}_{2}$ substrate.

Fabrication of samples: The metasurfaces were fabricated on a fused quartz substrate by utilizing electron beam lithography and including the processes such as deposition, patterning, lift-off and etching. First, a 350-nm-thick amorphous silicon $(\alpha-\mathrm{Si})$ film was deposited by plasma-enhanced chemical vapor deposition (PECVD) method at $120^{\circ} \mathrm{C}$, during which the flow rates of $\mathrm{SiH}_{4}$ and $\mathrm{Ar}$ are 10 and $475 \mathrm{sccm}$. And the deposition pressure and RF power are $650 \mathrm{mTorr}$ and $20 \mathrm{~W}$, respectively. Then a PMMA film of $300 \mathrm{~nm}$ was spinning coated and covered by PEDOT: PSS film as a conducting layer. The desired structure was patterned by utilizing JEOL 6300FS EBL at a base dose of $1000 \mu \mathrm{C} / \mathrm{cm}^{2}$ with an accelerating voltage of $100 \mathrm{kV}$ for 1 hour. After the exposure process, the conducting layer was washed away and the resist was developed in 1:3 MIBK: IPA solution for $40 \mathrm{~s}$ and rinsed in IPA for $30 \mathrm{~s}$ successively, followed by a deposition of $80 \mathrm{~nm} \mathrm{Cr}$ using electron beam evaporation deposition (EBD) method. To realize the lift-off process, the sample was immersed in hot acetone of $75^{\circ} \mathrm{C}$ and cleaned by ultrasonic. Finally, by using inductively coupled plasma (ICP) reactive ion etching (RIE) method with $\mathrm{HBr}$ at room temperature (RT) for $260 \mathrm{~s}$ (flow rate of $50 \mathrm{sccm}$, the pressure of $10 \mathrm{mTorr}$ RF and ICP power of 50 and $750 \mathrm{~W}$, respectively), the desired structure was transferred from $\mathrm{Cr}$ to silicon and the residual $\mathrm{Cr}$ was removed by cerium (IV) ammonium nitrate. The five planar chiral q-BIC metasurfaces are composed of $500 \times 500$ periods $(425 \mu \mathrm{m} \times 425 \mu \mathrm{m})$ with different cross-sections $(\delta=0 \mathrm{~nm}, 20 \mathrm{~nm}, 40 \mathrm{~nm}, 60 \mathrm{~nm}$, and $80 \mathrm{~nm})$, and the final structures are shown in Fig. 4a.

Characterization of samples: A supercontinuum laser (Fianium-WL-SC480) was employed as the broadband light source for the measurement of the transmission spectra of all polarization components under circular polarization incidences. Incident light with a circular polarization was generated by cascading a broadband polarizer and a quarter waveplate from the supercontinuum laser. Then, the incident light was focused on the sample by a lens with a focal length of $5 \mathrm{~cm}$. Subsequently, the polarization components are detected by a quarter waveplate and a polarizer. The transmittances were measured by means of an Ocean spectrometer (flame-NIR). Moreover, the sample was mounted on a rotation stage for varying incident angles in the measurement.

\section{Author Contributions}

Z.-L.D. and T.S. conceived the idea. T.S., Z.-L.D. and X.L. designed the experiments. T.S., Z.-L.D. carried out the design and simulation of the metasurface. T.S., Z.-L.D., G. H. A.O and Y. Z conducted the theoretical analysis of the results. G.G. and J.L. fabricated the samples. T.S. performed the measurements. Z.-L.D., Y.K., A.A. and X.L. supervised the project. Z.-L.D., T.S., and X.L. analyzed the data and wrote the manuscript. All authors contributed to the revision and discussion of the manuscript.

\section{Acknowledgements}

The authors acknowledge the funding support provided by National Key R\&D Program of China (2021YFB2802003), National Natural Science Foundation of China (NSFC) (62075084, 61522504, 61420106014, 12074420), Guangdong Basic and Applied Basic Research Foundation 

Guangzhou Science and Technology Program (202102020566), Guangdong Provincial Innovation and Entrepreneurship Project (2016ZT06D081), Beijing Municipal Science\&Technology Commission \&Administrative Commission of Zhongguancun Science Park (Z211100004821009), Air Force Office of Scientific Research, Vannevar Bush Faculty Fellowship, Simons Foundation, and Australian Research Council (grant DP210101292).

\section{Data availability}

The data that support the findings of this study are available from the corresponding author upon reasonable request.

\section{Conflict of Interest}

The authors declare no conflict of interest.

\section{References}

1. Chen, Y., Gao, J. \& Yang, X. Chiral Metamaterials of Plasmonic Slanted Nanoapertures with Symmetry Breaking. Nano Lett. 18, 520-527 (2018).

2. Plum, E., Fedotov, V. A. \& Zheludev, N. I. Extrinsic electromagnetic chirality in metamaterials. J. Opt. A: Pure Appl. Opt. 11, 074009 (2009).

3. Valev, V. K., Baumberg, J. J., Sibilia, C. \& Verbiest, T. Chirality and chiroptical effects in plasmonic nanostructures: fundamentals, recent progress, and outlook. Adv. Mater. 25, 25172534 (2013).

4. Le Bel, J. A. Sur les relations qui existent entre les formules atomiques des corps organiques et le pouvoir rotatoire de leurs dissolutions. Bull. Soc. Chim. Fr 22, 337-347 (1874).

5. Hazen, R. M. \& Sholl, D. S. Chiral selection on inorganic crystalline surfaces. Nat. Mater. 2 , 367-374 (2003).

6. Gansel, J. K., et al. Gold helix photonic metamaterial as broadband circular polarizer. Science 325, 1513-1515 (2009).

7. Mark, A. G., Gibbs, J. G., Lee, T. C. \& Fischer, P. Hybrid nanocolloids with programmed threedimensional shape and material composition. Nat. Mater. 12, 802-807 (2013).

8. Zhou, J. F., et al. Negative refractive index due to chirality. Phys. Rev. B 79, 121104 (2009).

9. Decker, M., et al. Strong optical activity from twisted-cross photonic metamaterials. Opt. Lett. 34, 2501-2503 (2009).

10. Zhang, S., et al. Photoinduced handedness switching in terahertz chiral metamolecules. Nat. Commun. 3, 942 (2012).

11. Menzel, C., et al. Asymmetric transmission of linearly polarized light at optical metamaterials. Phys. Rev. Lett. 104, 253902 (2010).

12. Hu, G., Wang, M., Mazor, Y., Qiu, C.-W. \& Alù, A. Tailoring light with layered and moiré metasurfaces. Trends Chem. 3, 342-358 (2021).

13. Fedotov, V. A., et al. Asymmetric propagation of electromagnetic waves through a planar chiral structure. Phys. Rev. Lett. 97, 167401 (2006).

14. Wu, C., et al. Spectrally selective chiral silicon metasurfaces based on infrared Fano resonances. Nat. Commun. 5, 3892 (2014). 
15. Zhu, A. Y., et al. Giant intrinsic chiro-optical activity in planar dielectric nanostructures. Light: Sci. Appl. 7, 17158 (2018).

16. Wang, S., et al. Arbitrary polarization conversion dichroism metasurfaces for all-in-one full Poincare sphere polarizers. Light: Sci. Appl. 10, 24 (2021).

17. Plum, E., Fedotov, V. A. \& Zheludev, N. I. Optical activity in extrinsically chiral metamaterial. Appl. Phys. Lett. 93, 191911 (2008).

18. Plum, E., et al. Metamaterials: optical activity without chirality. Phys. Rev. Lett. 102, 113902 (2009).

19. Sersic, I., van de Haar, M. A., Arango, F. B. \& Koenderink, A. F. Ubiquity of optical activity in planar metamaterial scatterers. Phys. Rev. Lett. 108, 223903 (2012).

20. Cao, T., Wei, C., Mao, L. \& Li, Y. Extrinsic 2D chirality: giant circular conversion dichroism from a metal-dielectric-metal square array. Sci. Rep. 4, 7442 (2014).

21. Chen, W., Yang, Q., Chen, Y. \& Liu, W. Extremize Optical Chiralities through Polarization Singularities. Phys. Rev. Lett. 126, 253901 (2021).

22. Chen, C., et al. Metasurfaces with Planar Chiral Meta-Atoms for Spin Light Manipulation. Nano Lett. 21, 1815-1821 (2021).

23. Zhang, F., et al. All-Dielectric Metasurfaces for Simultaneous Giant Circular Asymmetric Transmission and Wavefront Shaping Based on Asymmetric Photonic Spin-Orbit Interactions. Adv. Fun. Mater. 27, 1704295 (2017).

24. Ma, Z., et al. All-dielectric planar chiral metasurface with gradient geometric phase. Opt. Express 26, 6067-6078 (2018).

25. Chen, Y., Yang, X. \& Gao, J. Spin-controlled wavefront shaping with plasmonic chiral geometric metasurfaces. Light: Sci. Appl. 7, 84 (2018).

26. Wang, Q., et al. Reflective chiral meta-holography: multiplexing holograms for circularly polarized waves. Light: Sci. Appl. 7, 25 (2018).

27. Ha, S. T., et al. Directional lasing in resonant semiconductor nanoantenna arrays. Nat. Nanotechnol. 13, 1042-1047 (2018).

28. Kodigala, A., et al. Lasing action from photonic bound states in continuum. Nature 541, 196199 (2017).

29. Chen, Z. \& Segev, M. Highlighting photonics: looking into the next decade. eLight 1, 2 (2021).

30. Yesilkoy, F., et al. Ultrasensitive hyperspectral imaging and biodetection enabled by dielectric metasurfaces. Nat. Photonics 13, 390-396 (2019).

31. Leitis, A., et al. Angle-multiplexed all-dielectric metasurfaces for broadband molecular fingerprint retrieval. Sci. Adv. 5, eaaw2871 (2019).

32. Tittl, A., et al. Imaging-based molecular barcoding with pixelated dielectric metasurfaces. Science 360, 1105-1109 (2018).

33. Koshelev, K., et al. Subwavelength dielectric resonators for nonlinear nanophotonics. Science 367, 288-292 (2020).

34. Liu, Z., et al. High-Q Quasibound States in the Continuum for Nonlinear Metasurfaces. Phys. Rev. Lett. 123, 253901 (2019).

35. Koshelev, K., et al. Nonlinear Metasurfaces Governed by Bound States in the Continuum. ACS Photonics 6, 1639-1644 (2019).

36. Carletti, L., Koshelev, K., De Angelis, C. \& Kivshar, Y. Giant Nonlinear Response at the Nanoscale Driven by Bound States in the Continuum. Phys. Rev. Lett. 121, 033903 (2018). 
37. Fang, C., et al. High-Q resonances governed by the quasi-bound states in the continuum in alldielectric metasurfaces. Opto-Electron. Adv. 4, 200030-200030 (2021).

38. Gandolfi, M., Tognazzi, A., Rocco, D., De Angelis, C. \& Carletti, L. Near-unity third-harmonic circular dichroism driven by a quasibound state in the continuum in asymmetric silicon metasurfaces. Phys. Rev. A 104, 023524 (2021).

39. Koshelev, K., Lepeshov, S., Liu, M., Bogdanov, A. \& Kivshar, Y. Asymmetric Metasurfaces with High-Q Resonances Governed by Bound States in the Continuum. Phys. Rev. Lett. 121, 193903 (2018).

40. Shi, T., Deng, Z.-L., Tu, Q.-A., Cao, Y. \& Li, X. Displacement-mediated bound states in the continuum in all-dielectric superlattice metasurfaces. PhotoniX 2, 7 (2021).

41. Liu, M. \& Choi, D. Y. Extreme Huygens' Metasurfaces Based on Quasi-Bound States in the Continuum. Nano Lett. 18, 8062-8069 (2018).

42. Overvig, A. C., Malek, S. C., Carter, M. J., Shrestha, S. \& Yu, N. Selection rules for quasibound states in the continuum. Phys. Rev. B 102, 035434 (2020).

43. Hsu, C. W., et al. Observation of trapped light within the radiation continuum. Nature 499, 188191 (2013).

44. Zhen, B., Hsu, C. W., Lu, L., Stone, A. D. \& Soljacic, M. Topological nature of optical bound states in the continuum. Phys. Rev. Lett. 113, 257401 (2014).

45. Hsu, C. W., Zhen, B., Stone, A. D., Joannopoulos, J. D. \& Soljačić, M. Bound states in the continuum. Nat. Rev. Mater. 1, 1-13 (2016).

46. Rybin, M. V., et al. High-Q Supercavity Modes in Subwavelength Dielectric Resonators. Phys. Rev. Lett. 119, 243901 (2017).

47. Doeleman, H. M., Monticone, F., den Hollander, W., Alù, A. \& Koenderink, A. F. Experimental observation of a polarization vortex at an optical bound state in the continuum. Nat. Photonics 12, 397-401 (2018).

48. Jin, J., et al. Topologically enabled ultrahigh-Q guided resonances robust to out-of-plane scattering. Nature 574, 501-504 (2019).

49. Zeng, Y., Hu, G., Liu, K., Tang, Z. \& Qiu, C. W. Dynamics of Topological Polarization Singularity in Momentum Space. Phys. Rev. Lett. 127, 176101 (2021).

50. Gorkunov, M. V., Antonov, A. A. \& Kivshar, Y. S. Metasurfaces with Maximum Chirality Empowered by Bound States in the Continuum. Phys. Rev. Lett. 125, 093903 (2020).

51. Overvig, A., Yu, N. \& Alù, A. Chiral Quasi-Bound States in the Continuum. Phys. Rev. Lett. 126, 073001 (2021).

52. Overvig, A. C., Mann, S. A. \& Alù, A. Thermal Metasurfaces: Complete Emission Control by Combining Local and Nonlocal Light-Matter Interactions. Phys. Rev. X 11, 021050 (2021).

53. Overvig, A. \& Alù, A. Wavefront-selective Fano resonant metasurfaces. Adv. Photonics 3, 026002 (2021).

54. Overvig, A. C., Malek, S. C. \& Yu, N. Multifunctional Nonlocal Metasurfaces. Phys. Rev. Lett. 125, 017402 (2020).

55. Dixon, J., Lawrence, M., Barton, D. R. \& Dionne, J. Self-Isolated Raman Lasing with a Chiral Dielectric Metasurface. Phys. Rev. Lett. 126, 123201 (2021).

56. Kim, K. H. \& Kim, J. R. High - Q Chiroptical Resonances by Quasi - Bound States in the Continuum in Dielectric Metasurfaces with Simultaneously Broken In - Plane Inversion and Mirror Symmetries. Adv. Opt. Mater. 9, 2101162 (2021). 
57. Liu, W., et al. Circularly Polarized States Spawning from Bound States in the Continuum. Phys.

Rev. Lett. 123, 116104 (2019).

58. Gorkunov, M. V., Antonov, A. A., Tuz, V. R., Kupriianov, A. S. \& Kivshar, Y. S. Bound States in the Continuum Underpin Near-Lossless Maximum Chirality in Dielectric Metasurfaces. Adv. Opt. Mater. 9, 2100797 (2021).

59. Menzel, C., Rockstuhl, C. \& Lederer, F. Advanced Jones calculus for the classification of periodic metamaterials. Phys. Rev. A 82, 053811 (2010).

60. Kenney, M., et al. Pancharatnam-Berry Phase Induced Spin-Selective Transmission in Herringbone Dielectric Metamaterials. Adv. Mater. 28, 9567-9572 (2016).

61. Liu, V. \& Fan, S. S4 : A free electromagnetic solver for layered periodic structures. Comput. Phys. Commun. 183, 2233-2244 (2012). 

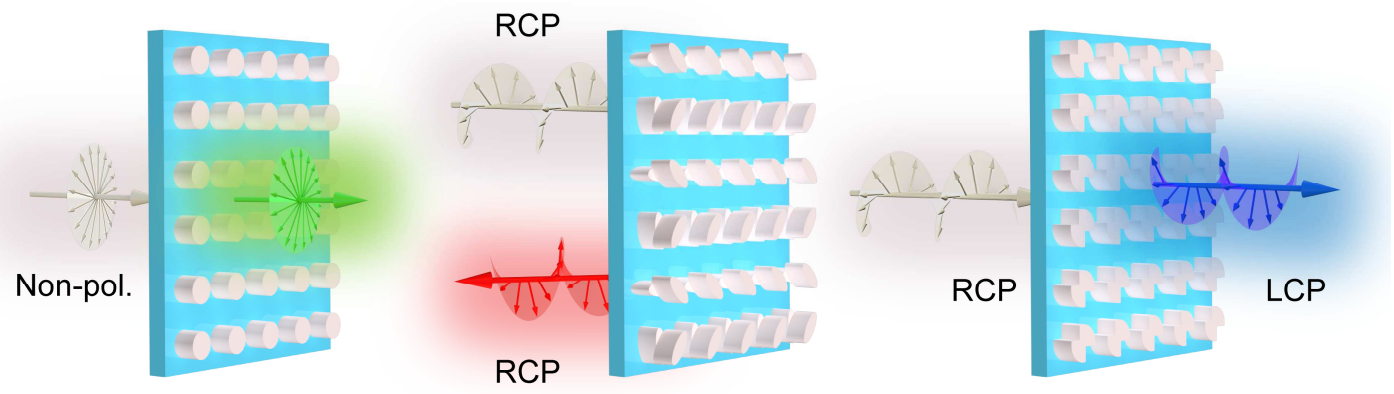

d

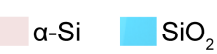

e

f
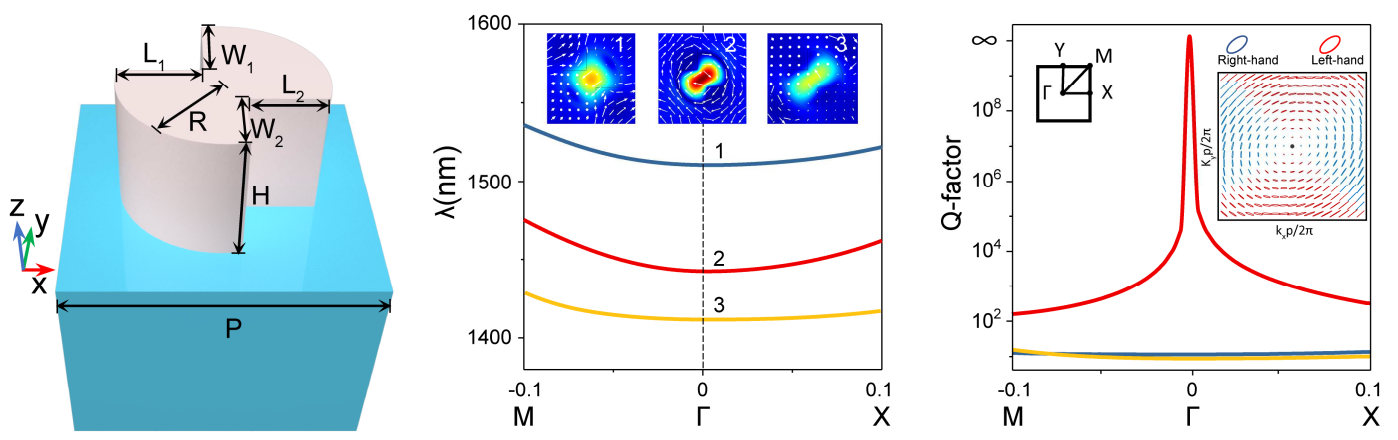

Figure 1. Schematics of different BIC states and planar chiral q-BIC metasurface with a polarization singularity enclosed by vortex elliptical eigen-polarizations with non-vanishing helicities. (a-c) Comparison of q-BICs (a) in planar metasurfaces without polarization effect, (b) in stereo-nanostructures with 3D chiral effect in reflection, and (c) in planar metasurfaces with 2D chiral effect in transmission. (d) Schematic of the unit cell of the planar chiral q-BIC metasurface formed by the amorphous silicon ( $\alpha$-Si) DSS structure on a $\mathrm{SiO}_{2}$ substrate with parameters $P=850$ $\mathrm{nm}, R=280 \mathrm{~nm}, L_{1}=L_{2}=220 \mathrm{~nm}, W_{l}=W_{2}=191 \mathrm{~nm}$, and $H=350 \mathrm{~nm}$. (e) Band structure with real parts of the eigen-wavelength of the DSS metasurface near the $\Gamma$ point. Insets show the field patterns (color: magnetic distributions, arrow: electric vectors) of the tree typical modes. (f) Q-factors of the eigenmode in the $k$-space in the vicinity of the $\Gamma$ point. Insets show the first Brillouin zone and eigen-polarization profiles. On the eigen-polarization profiles, the polarization states are represented by ellipses of which the red and blue represent the eigen left-handed states and right-handed states, 
a

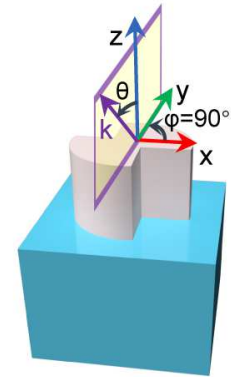

b

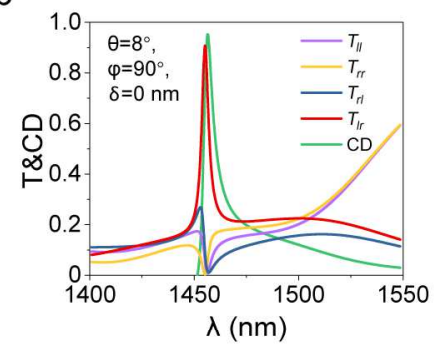

e

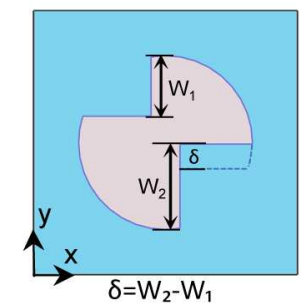

f

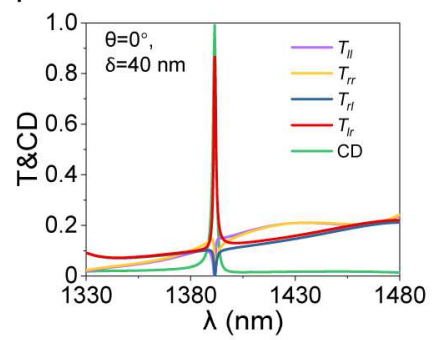

C

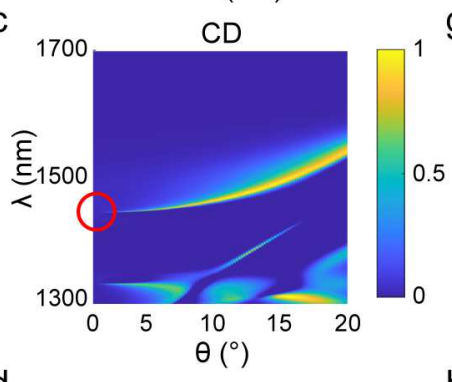

d

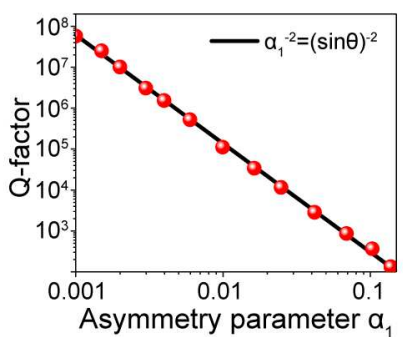

$g_{1500} \quad C D$

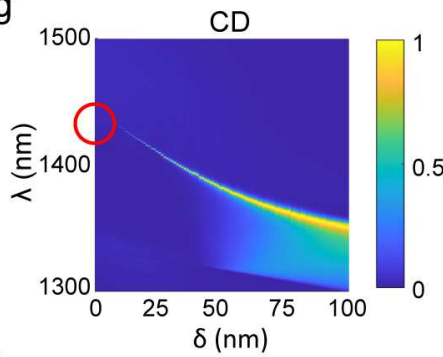

h

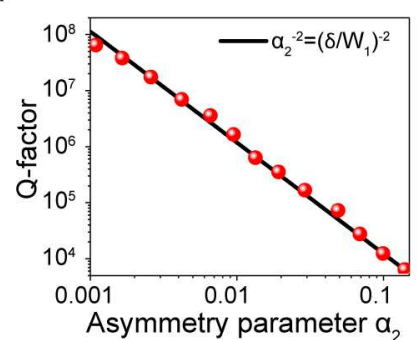

Figure 2. Proposed implementation of the DSS structure with both the extrinsic and intrinsic planar chirality by transferring the BIC to q-BICs. (a, e) Schematic of the symmetry-breaking processes that transfer the BIC to planar chiral q-BICs: (a) illumination symmetry breaking by varying incident angle $\theta$, (e) in-plane geometry symmetry breaking by $\delta=W_{2}-W_{l}$. (b, f) Simulated transmission Jones matrix spectra of $T_{l l}, T_{r r}, T_{r l}$ and $T_{l r}$ as well as the CD spectrum of the metasurface with (b) the same parameters as Fig. $1 \mathrm{~d}(\delta=0 \mathrm{~nm})$ under oblique incidence $\left(\theta=8^{\circ}, \varphi=90^{\circ}\right)$ and with (f) an asymmetric structure parameter $(\delta=40 \mathrm{~nm})$ for normal incidences. (c, g) The evolution of CD spectra by continuous varying (c) incident angle $\theta$ along the $\varphi=90^{\circ}$ direction and by (g) the geometry asymmetry parameter $\delta .(\mathrm{d}, \mathrm{h})$ Dependence of the Q-factors of the planar chiral q-BIC mode on the relative asymmetry parameter (d) $\alpha_{l}=\sin \theta$, (h) $\alpha_{2}=\delta / W_{l}$ around the chiral q-BIC state. The solid line shows an inverse quadratic fitting. 
a

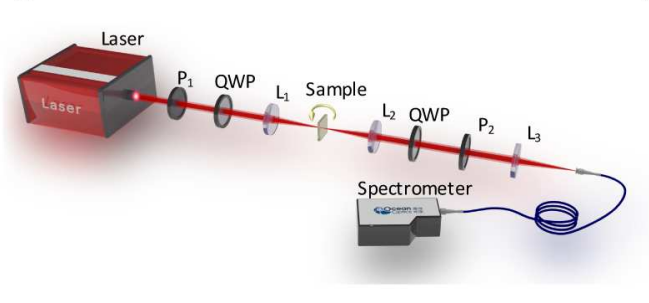

C

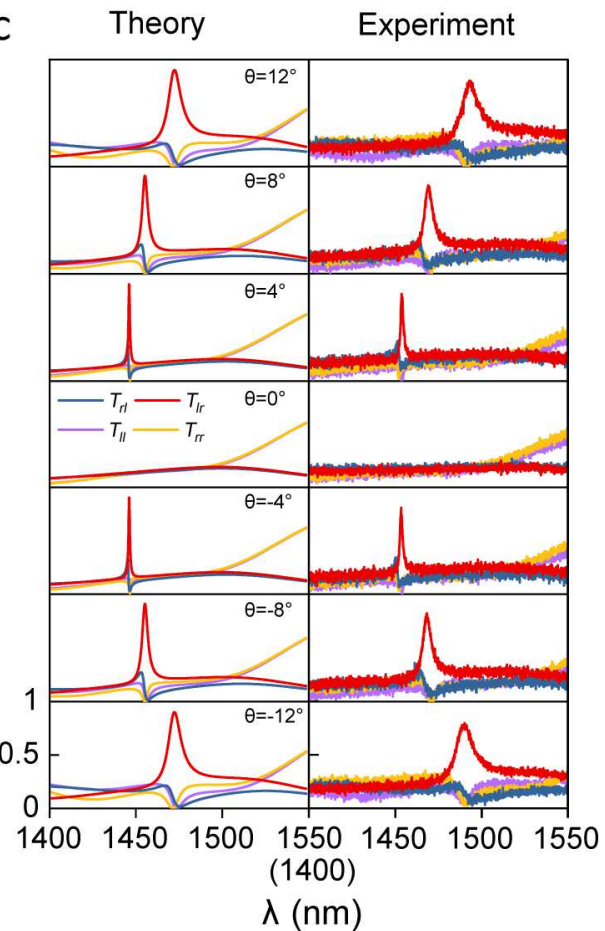

b

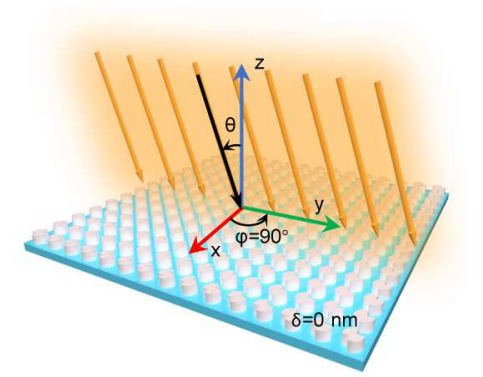

d

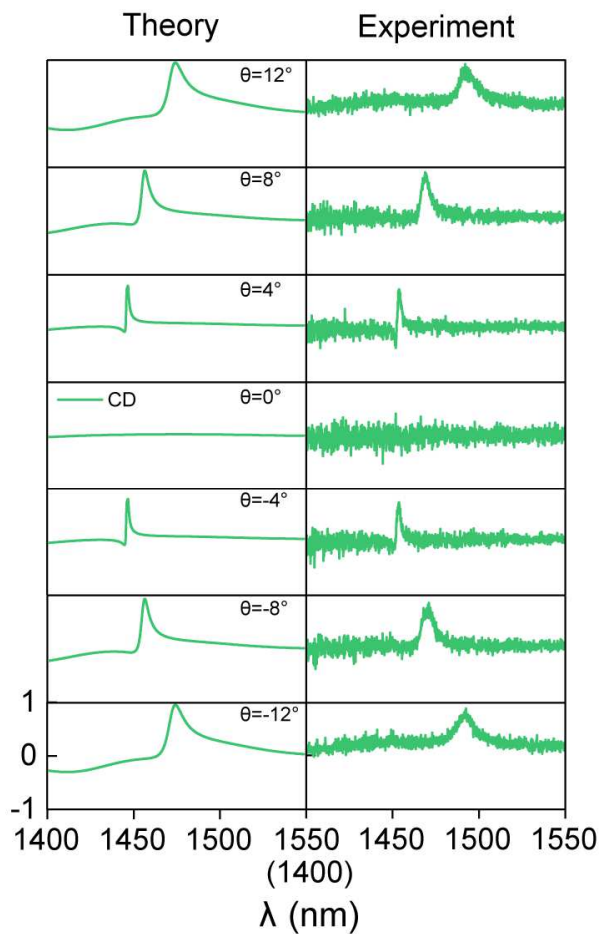

474

475

476

477

478

479

480

481

482

Figure 3. Experimental verification of the extrinsic planar chiral q-BIC with illumination symmetry breaking. (a) Experimental setup for the Jones matrix spectra measurement under the circular polarization basis. $\mathrm{P}_{1}$ and $\mathrm{P}_{2}$ represent the polarizers, $\mathrm{QWP}$ is the quarter-wave plate, $\mathrm{L}_{1}, \mathrm{~L}_{2}$, and $\mathrm{L}_{3}$ are lenses. (b) Schematic of the metasurface with $\delta=0$ supporting chiral q-BICs through different incident angles along the $\varphi=90^{\circ}$ direction. (c) Simulated and measured transmission Jones matrix spectra $\left(T_{l r}, T_{r l}, T_{r r}\right.$ and $\left.T_{l l}\right)$ for different incident angles $\left(\theta=0^{\circ}, \pm 4^{\circ}, \pm 8^{\circ}, \pm 12^{\circ}\right)$ along the $\varphi=90^{\circ}$ direction. (d) Simulated and measured CD spectra for different incident angles extracted from the Jones matrix spectra in (c). 
a

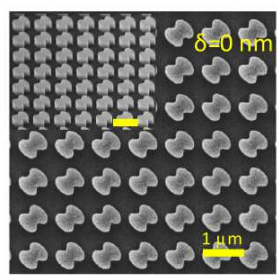

b
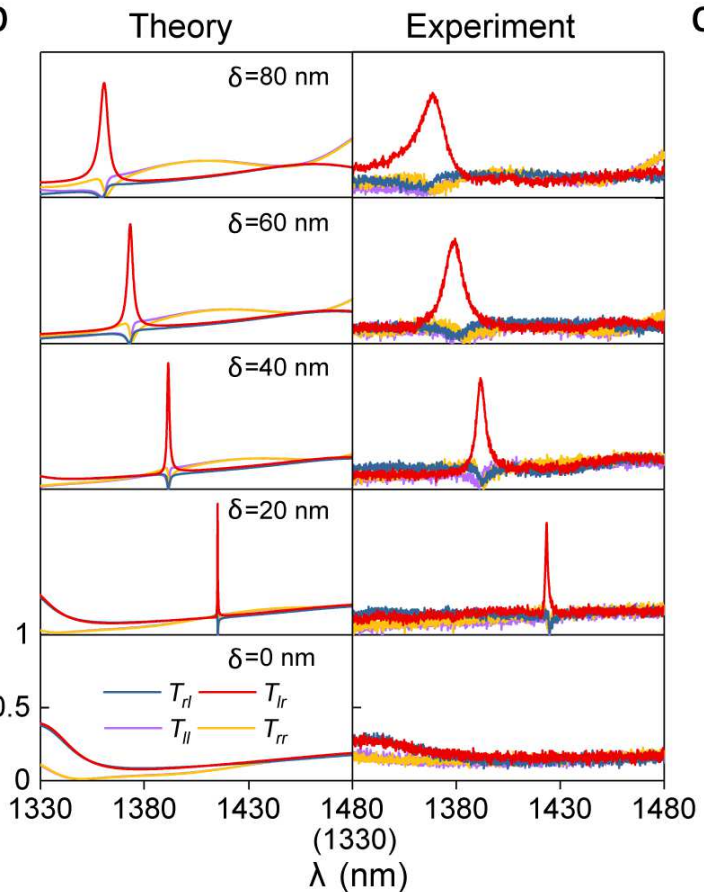
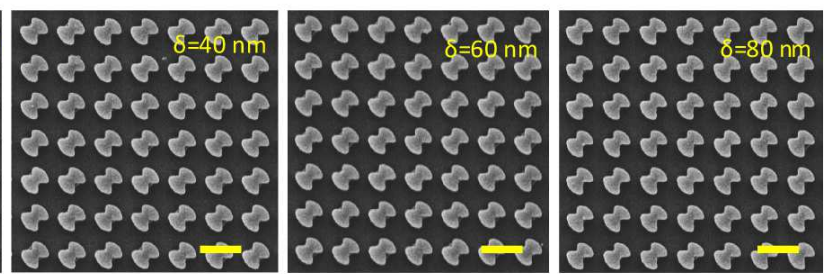

C

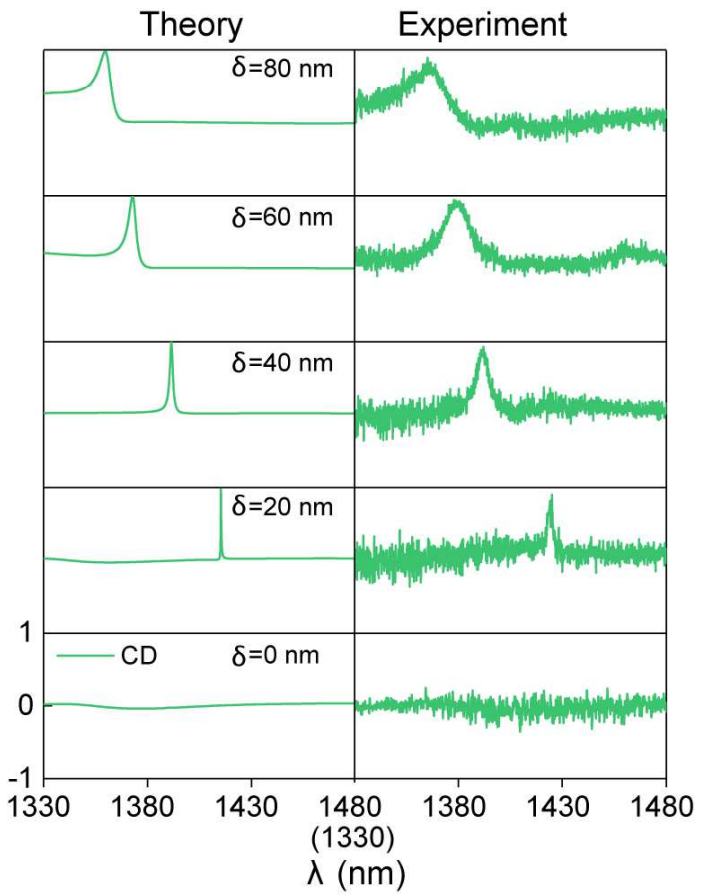

Figure 4. Experimental verification of the intrinsic planar chiral q-BIC with various in-plane geometry asymmetry parameters. (a) The top view of the SEM image of five fabricated DSS metasurfaces with asymmetry parameters $\delta=0 \mathrm{~nm}, 20 \mathrm{~nm}, 40 \mathrm{~nm}, 60 \mathrm{~nm}$, and $80 \mathrm{~nm}$, respectively. The inset shows the slant view of the metasurface. Scale bar: $1 \mu \mathrm{m}$. (b) Simulated and measured transmission Jones matrix spectra $\left(T_{l r}, T_{r l}, T_{r r}\right.$ and $\left.T_{l l}\right)$ of the five DSS metasurface samples with different $\delta$. (c) Simulated and measured CD spectra for different $\delta$ extracted from the Jones matrix spectra in (b). 


\section{Supplementary Files}

This is a list of supplementary files associated with this preprint. Click to download.

- PlanarchiralqBICSINC.pdf 